\title{
Detection of severe acute respiratory syndrome Coronavirus- 2 in the tears of patients with Coronavirus disease 2019
}

\author{
Saeed Karimi ${ }^{1,2} \cdot$ Amir Arabi $i^{1,2} \cdot$ Toktam Shahraki ${ }^{1,2} \cdot$ Sare Safi $\mathbb{i}^{3}$ \\ Received: 17 April 2020 / Revised: 1 May 2020 / Accepted: 6 May 2020 / Published online: 18 May 2020 \\ (c) The Author(s), under exclusive licence to The Royal College of Ophthalmologists 2020
}

\begin{abstract}
Objectives Since there are few reports on the ocular involvement of coronavirus disease 2019 (COVID-19) patients, this study aimed to assess the presence of severe acute respiratory syndrome Coronavirus-2 (SARS-CoV-2) in the tears of patients with COVID-19.

Methods In this prospective case series, nasopharyngeal and tear sampling of 43 patients with severe COVID-19 were performed. The quantitative reverse transcription polymerase chain reaction (RT-PCR) was conducted to detect SARS-CoV2. Ocular and systemic signs and symptoms were recorded from their medical history.

Results The mean age of patients was $56 \pm 13$ years. The average disease time from initiation of symptoms was 3.27 days, range: 1-7 days. Forty-one patients (95.3\%) had fever at the time of sampling. Only one patient had conjunctivitis. Thirty (69.8\%) nasopharyngeal and three (7\%) tear samples were positive for SARS-CoV-2. The result of tear sample was positive in the patient with conjunctivitis. All patients with positive tear RT-PCR results had positive nasopharyngeal RT-PCR results.

Conclusions Ocular manifestation was rare in this series of severe COVID-19 patients, however, 7\% of the patients had viral RNA in their conjunctival secretions. Therefore, possibility of ocular transmission should be considered even in the absence of ocular manifestations.
\end{abstract}

\section{Introduction}

Severe acute respiratory syndrome coronavirus 2 (SARS$\mathrm{CoV}-2$ ) is a novel member of the betacoronavirus genus which has caused the ongoing pandemic of coronavirus disease 2019 (COVID-19). Fever and respiratory symptoms, including cough and shortness of breath have been reported to be the most common manifestations of the disease [1]. The signs and symptoms often manifest within 2 to 14 days following primary exposure [1]. SARS-CoV-2 is highly contagious and can be transmitted through

Amir Arabi

amir_arab_91@yahoo.com

1 Ophthalmic Research Centre, Shahid Beheshti University of Medical Sciences, Tehran, Iran

2 Department of Ophthalmology, Torfeh Medical Centre, Shahid Beheshti University of Medical Sciences, Tehran, Iran

3 Ophthalmic Epidemiology Research Centre, Shahid Beheshti University of Medical Sciences, Tehran, Iran respiratory droplets and contact with infected persons or fomites. Aerosol transmission has not been confiremd [2].

Some clinicians have expressed concerns about the transmission of SARS-CoV-2 via tears and conjunctival secretions of infected patients [3]. A genetic similarity of greater than $80 \%$ have been reported between SARS-CoV and SARS-CoV-2 [4]. During the SARS outbreak in 2004, some studies reported the presence of SARS-CoV in the tears of the patients [5]. Additionally, during the recent outbreak of the coronavirus 2019, a large number of ophthalmologists have been reported to be infected [2]. Some recent studies have evaluated the infectivity of tears of COVID-19 patients [3]. In the present study we evaluated the presence of SARS-CoV-2 in tears of patients diagnosed with clinically confirmed COVID-19.

\section{Materials and methods}

In this prospective interventional case series, we included patients with clinically confirmed COVID-19 patients who were admitted to our hospital between March 28, 2020 and 
April 5, 2020. The study protocol was approved by institutional ethics committee and followed the Declaration of Helsinki. A written informed consent was provided from each patient or patient's family.

The criteria for clinical diagnosis and admission of COVID-19 patients (severe COVID-19) followed the Iran National Guideline on Prevention and Control of COVID19: (1) highly suspicious radiological findings related to COVID-19 (unilateral or bilateral multi-lobar infiltration of the lungs especially the peripheral zones and/or ground glass appearance) as confirmed by a radiologist; (2) pneumonia with a poor response to appropriate treatment and an unpredicted progression; (3) respiratory rate $\geq 24$ cycles/minute, oxygen saturation $\leq 90 \%$, or heart rate $>125$ beats/min.

Quantitative reverse transcription polymerase chain reaction (RT-PCR) tests were performed on the first day of admission for each patient. Sampling of both nasopharyngeal and conjunctival secretions was performed at the same time for each patient with caution to avoid any cross contamination. RT-PCR assay sampling of nasopharyngeal secretions was performed according to the protocol established by the World Health Organization (WHO). To collect tear samples, we used disposable swabs. After opening the eyelids, the swab was placed in the lower fornix for at least $10 \mathrm{~s}$ in each eye. No anesthesia was used during the procedure. All tear samples were collected by a single ophthalmologist (AA). All nasopharyngeal and tear specimens were stored at $4{ }^{\circ} \mathrm{C}$ and were immediately sent to the same laboratory for processing.

\section{Results}

A total of 43 patients (14 female and 29 male) were enrolled. The mean age of the patients was $56 \pm 13$ years. At the time of sampling, the average disease time from the initiation of symptoms was 3.27 days, range 1-7 days. The mean of body temperature was $38.33{ }^{\circ} \mathrm{C}$. At the time of sampling, 41 (95.3\%), 38 (88.3\%), and $30(69.8 \%)$ patients had fever, shortness of breath, and cough, respectively. Other symptoms were myalgia, fatigue, and chills. Nine patients $(20.9 \%)$ were admitted to ICU because of respiratory failure needing ventilators. One patient had bilateral conjunctivitis that was manifested by conjunctival congestion and mucus discharge. In addition, one patient reported bilateral foreign body sensation since 3 days before the onset of systemic manifestations. Demographic and clinical characteristics of the study patients are summarized in Table 1.

Nasopharyngeal RT-PCR yielded positive results in 30 patients $(69.8 \%)$. Both tear RT-PCR and nasopharyngeal RT-PCR were positive for SARS-CoV-2 in $3(7 \%)$ patients.
Table 1 Demographic and clinical characteristics of the study patients.

\begin{tabular}{ll}
\hline Parameter & Value \\
\hline Age (Mean \pm SD) & $56.6 \pm 13.7$ \\
Sex & $29(67.5 \%)$ \\
$\quad$ Male $(\%)$ & $14(32.5 \%)$ \\
$\quad$ Female $(\%)$ & 3.27 days $(1$ to 7 days) \\
Disease time on the day of admission & \\
(Range) & \\
Systemic signs and symptoms & $38.33{ }^{\circ} \mathrm{C}$ \\
$\quad$ Body temperature (Mean) & $41(95.3 \%)$ \\
$\quad$ Fever (\%) & $38(88.3 \%)$ \\
$\quad$ Shortness of breath (\%) & $30(69.8 \%)$ \\
$\quad$ Cough (\%) & $1(2.3 \%)$ \\
Ocular findings & $1(2.3 \%)$ \\
$\quad$ Conjunctivitis $(\%)$ &
\end{tabular}

SD Standard deviation.

Table 2 Nasopharyngeal and tear reverse transcription polymerase chain reaction tests results in clinically diagnosed COVID-19 patients.

\begin{tabular}{llll}
\hline Test & Positive & Negative & Total \\
\hline Nasopharyngeal RT-PCR & 30 & 13 & 43 \\
Tear RT-PCR & 3 & 40 & 43 \\
\hline
\end{tabular}

$R T-P C R$ Reverse transcription polymerase chain reaction.

None of these three patients were admitted to ICU. Tear RT-PCR was positive in the patient with conjunctivitis but negative for the patient with ocular foreign body sensation. Both tear RT-PCR and nasopharyngeal RT-PCR were negative in 13 patients (30\%). The results of RT-PCR tests are summarized in Table 2.

\section{Discussion}

Although the evidence of coronavirus transmission via ocular secretions has not been confirmed, in the present study we found 3 patients with positive tear RT-PCR test for SARS-CoV-2. The presence of SARS-CoV-2 in tears can be due to direct inoculation, migration of the virus from the respiratory tract through the nasolacrimal duct, or infection of the lacrimal gland and consequent shedding of the virus [6]. In addition, it has been postulated that the affinity of SARS-CoV to angiotensin-convertingenzyme-2 makes it possible for the virus to infect ocular tissues [7, 8].

The first case of conjunctivitis related to COVID-19 was reported by the Chinese pulmonary specialist. He claimed that he was infected via ocular secretions [3]. During the recent coronavirus pandemic, there have been both clinical 
Table 3 Summary of studies on conjunctival swab reverse transcription polymerase chain reaction results in COVID-19 patients.

\begin{tabular}{lllcc}
\hline Study & Sample size & Enrolled patients & Ocular signs and symptoms & SARS-CoV-2 detection in tear samples \\
\hline Wu et al. [13] & 38 & Clinically confirmed patients & $31.6 \%(12$ of 38$)$ & 2 of $38(5 \%)$ \\
Xia et al. [2] & 30 & Laboratory confirmed patients & $3 \%(1$ of 30$)$ & 1 of $30(3 \%)$ \\
Seah et al. [10] & 17 & Laboratory confirmed patients & $5 \%(1$ of 17$)$ & 0 of $17(0 \%)$ \\
Sun et al. [12] & 72 & Laboratory confirmed patients & $2.7 \%(2$ of 72$)$ & 1 of $72(1.3 \%)$ \\
Deng et al. [14] & 114 & Clinically confirmed patients & $0 \%(0$ of 114$)$ & 0 of $114(0 \%)$ \\
Zhou et al. [11] & 67 & Confirmed or suspected patients & $1.4 \%(1$ of 67$)$ & $4.4 \%(3$ of 67$)$ \\
\hline
\end{tabular}

and laboratory reports of ocular involvement (Table 3). In a large study in China, conjunctival congestion was reported in 9 out of 1099 patients with laboratory-confirmed disease [9]. According to the studies in which tear sampling has been performed, a total of 17 patients with COVID-19 have had ocular manifestations including conjunctivitis, tearing, and foreign body sensation.

In two case series that have evaluated the presence of SARS-CoV-2 RNA in conjunctival secretions through RTPCR, no positive result has been reported. In a study in Singapore, multiple tear samples were collected from 17 cases between day 3 to day 20 following the incidence of the disease [10]. None of the 64 RT-PCR reports was positive for SARS-CoV-2 RNA. Similarly, in another study on 114 COVID-19 patients in China, all ocular samples were negative for SARS-CoV-2 RNA. On the other hand, there have been reports of positive RT-PCR results of conjunctival swab samples. In a study by Xia et al., 30 COVID-19 patients were enrolled, and the tear sample of 1 patient yielded positive RT-PCR result [2]. In another study on 67 patients, conjunctival swab samples from 1 patient yielded positive RT-PCR result, and 2 other patients had probable positive reports [11]. Sun et al. studied 72 COVID-19 cases and reported that SARS-CoV-2 was detected in the conjunctival samples of only one patient who was an emergency department nurse [12]. In another case series of 38 COVID-19 patients, 12 patients were reported to have ocular manifestations, but only two of them had positive tear RT-PCR results [13].

Our study showed a slightly higher rate of positive tear RT-PCR assay for SARS-CoV-2 than the previous studies. This may be explained as follows. All of our patients were admitted to the hospital and had positive findings on chest computed tomography. We performed sampling on the first day of admission when the viral load seems to be high, and the sampling swabs were in contact with the tear for a longer duration (10 s) than that reported previously. Racial variation and differences may be important. Furthermore, it may be related to the variety of the virus subtypes in different regions.

In this study, RT-PCR of nasopharyngeal samples was positive in $69.8 \%$ of clinically diagnosed COVID-19 patients. All 3 cases (7\%) who were positive for tear RT-PCR, were positive for nasopharyngeal RT-PCR as well. There was no tear positive cases among the nasopharyngeal negative cases. This shows that the viral load of nasopharynx is probably higher than that of tears. The prevalence of ocular manifestations such as conjunctivitis was low in our patients. One patient had bilateral conjunctivitis, and another one had foreign body sensation. Tear RT-PCR was positive for the patient with conjunctivitis and was negative for patient with foreign body sensation. Therefore, among the three patients with positive tear RT-PCR test, only one patient had ocular manifestations (conjunctivitis) and the other two patients had no oculars signs or symptoms. This suggests that the tears of patients with COVID-19 may be infective despite the absence of ocular manifestations.

RT-PCR may have some limitations in detecting viral infection of ocular samples. Small quantities of viral RNA in conjunctival secretions in addition to a probable short duration of viral shedding in tears may contribute to falsenegative reports in RT-PCR [14]. Difficulty in collecting adequate amount of tear sample is another problem. Moreover, the time of sampling in the course of the disease may affect the test results.

Our study was limited due to the small sample size, enrollment of the patients from a single medical center, and one-time sampling from patients (only on the first day of admission). Future multi-center studies with a large sample size and multiple sampling of tears during the disease course in each patient are recommended.

In conclusion, this study showed a higher rate of SARSCoV-2 virus shedding (7\%) in tears of COVID-19 patients than previous reports. The possibility of disease transmission through tears should be considered even in the absence of ocular manifestations.

\section{Summary}

\section{What was known before}

- There are few reports on the ocular involvement of coronavirus disease 2019 (COVID-19) patients. 


\section{What this study adds}

- Ocular manifestation was rare in severe COVID-19 patients, however, $7 \%$ of the patients had viral RNA in their conjunctival secretions. Therefore, possibility of ocular transmission should be considered even in the absence of ocular manifestations.

Acknowledgements The study was supported by the team of physicians, laboratory staff, and nursing of Torfeh Medical Center.

\section{Compliance with ethical standards}

Conflict of interest The authors declare that they have no conflict of interest.

Publisher's note Springer Nature remains neutral with regard to jurisdictional claims in published maps and institutional affiliations.

\section{References}

1. McMichael TM, Currie DW, Clark S, Pogosjans S, Kay M, Schwartz NG, et al. Epidemiology of Covid-19 in a long-term care facility in King County, Washington. N Engl J Med. 2020. https://doi.org/10.1056/NEJMoa2005412.

2. Xia J, Tong J, Liu M, Shen Y, Guo D Evaluation of coronavirus in tears and conjunctival secretions of patients with SARS-CoV-2 infection. J Med Virol. 2020. https://doi.org/10.1002/jmv.25725.

3. Lu C-W, Liu X-F, Jia Z-F. 2019-nCoV transmission through the ocular surface must not be ignored. Lancet. 2020;395:e39.

4. Chan J, Kok K-H, Zheng Z, Chu H, To KK, Yuan S, et al. Genomic characterization of the 2019 novel human-pathogenic coronavirus isolated from a patient with atypical pneumonia after visiting Wuhan. Emerg Microbes Infect. 2020;9:221-36.

5. Loon SC, Teoh S, Oon L, Se-Thoe SY, Ling AE, Leo YS, et al. The severe acute respiratory syndrome coronavirus in tears. Br J Ophthalmol. 2004;88:861-3.

6. Seah I, Agrawal R. Can the coronavirus disease 2019 (COVID-19) affect the eyes? A review of coronaviruses and ocular implications in humans and animals. Ocul Immunol Inflamm. 2020;28:3915.

7. Wan Y, Shang J, Graham R, Baric R, Li F. Receptor recognition by novel coronavirus from Wuhan: an analysis based on decadelong structural studies of SARS. J Virol. 2020;94. pii: e00127-20. https://doi.org/10.1128/JVI.00127-20.

8. Holappa M, Vapaatalo H, Vaajanen A. Many faces of reninangiotensin system-focus on eye. Open Ophthalmol J. 2017;11: $122-42$.

9. Guan W-J, Ni Z-y, Hu Y, Liang WH, Ou CQ, He JX, et al. Clinical characteristics of 2019 novel coronavirus infection in China. N Engl J Med. 2020. https://doi.org/10.1056/NEJMoa2002032.

10. Seah I, Anderson D, Kang A, Wang L, Rao P, Young BE, et al. Assessing viral shedding and infectivity of tears in Coronavirus disease 2019 (COVID-19) patients. Ophthalmology. 2020. https://doi.org/10.1016/j.ophtha.2020.03.026.

11. Zhou Y, Zeng Y, Tong Y, Chen C. Ophthalmologic evidence against the interpersonal transmission of 2019 novel Coronavirus through conjunctiva. medRxi 2020. https://doi.org/10.1101/2020. 02.11.20021956.

12. Sun C, Wang Y, Liu G, Liu Z. Role of the eye in transmitting human coronavirus: what we know and what we do not know. Front Public Health. 2020;8:155. https://doi.org/10.3389/fpubh. 2020.00155.

13. Wu P, Duan F, Luo C, Liu Q, Qu X, Liang L, et al. Characteristics of ocular findings of patients with coronavirus disease 2019 (COVID-19) in Hubei Province, China. JAMA Ophthalmol. 2020. https://doi.org/10.1001/jamaophthalmol.2020.1291.

14. Deng C, Yang Y, Chen H, Chen W, Chen Z, Ma K, et al. Ocular detection of SARS-CoV-2 in 114 cases of COVID-19 pneumonia in Wuhan, China: an observational study. 2020. https://doi.org/10. 2139/ssrn.3543587. 